\title{
eJRIEPS
}

Ejournal de la recherche sur l'intervention en éducation physique et sport

$21 \mid 2010$

Varia

\section{Étude de cas des ressentis émotionnels de deux enseignantes débutantes d'éducation physique et sportive}

\section{Marina Loubet}

\section{(2) OpenEdition}

1 Journals

Édition électronique

URL : http://journals.openedition.org/ejrieps/4749

ISSN : 2105-0821

Éditeur

ELLIADD

Référence électronique

Marina Loubet, «Étude de cas des ressentis émotionnels de deux enseignantes débutantes d'éducation physique et sportive », eJRIEPS [En ligne], 21 | 2010, mis en ligne le 01 juillet 2010, consulté le 27 février 2020. URL : http://journals.openedition.org/ejrieps/4749

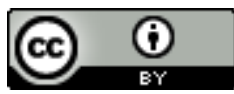

La revue eJRIEPS est mise à disposition selon les termes de la Creative Commons Attribution 4.0 International License. 
Etude de cas des ressentis émotionnels de deux enseignantes débutantes

d'éducation physique et sportive

Marina Loubet

UFR STAPS, Université de Montpellier I (Laboratoire SANTESIH - santé, éducation et situations de handicap)

\section{Résumé}

Cet article est consacré à l'étude clinique des émotions de deux enseignantes débutantes d'éducation physique et sportive. Le but de cette étude est de mettre en avant les origines des émotions ressenties au cours de l'année de stage. Les protocoles utilisés sont l'autosupervision et le récit de vie. Les principaux résultats mettent en avant que le fait d'enseigner l'EPS engendre des émotions à majorité ressenties comme "négatives" et propres à cette matière. Leur apparition cyclique révèle l'importance de certaines caractéristiques des activités sportives enseignées et suggère l'effet angoissant de la coupure des vacances.

Le travail présenté prolongeant d'autres recherches dans le monde de l'ergonomie (Cossette, 1999; Gendron, 2006) s'intéresse aux ressentis émotionnels d'enseignantes d'Éducation Physique et Sportive (EPS) débutantes en France. Au fil de l'année scolaire après avoir réussi un concours difficile (1/10 à 1/30 succès, selon le concours et les années) mais relativement théorique, l'enseignant stagiaire va découvrir "des responsabilités, des obligations et des difficultés qui se rattachent à l'exercice d'un métier" (Lamotte et Nerin, 2003). Nombreux sont les auteurs mettant en avant la difficulté d'entrer dans le métier d'enseignant en début de carrière de par la complexité de l'expérience en classe (Ria, 2004), la complexité de l'acte d'enseignement (Doyle, 1986; Roux-Perez, 2006), la difficulté supposée ou réelle de certains lieux de stage compte tenu de la population accueillie (Livret National du professeur stagiaire, 2002).

Lamour (1986) prétend que nous entrons tous dans le monde avec notre affectivité. Le jour de la rentrée scolaire les émotions sont multiples et elles vont être prédominantes tout au long de cette année de formation voire se prolonger durant la carrière. Tantôt proches du plaisir, tantôt proches de l'angoisse, les émotions ressenties par le Professeur des Lycées et Collèges $2^{\text {ème }}$ Année (désormais PLC2) vont être multiples, variées mais surtout 


\section{eJRIEPS 21 juillet 2010}

omniprésentes dans son apprentissage du métier d'enseignant d'EPS. Les termes utilisés pour caractériser les premiers pas dans le monde de l'enseignement sont relatifs à l'affect mais ressentis plutôt comme perturbateurs, dérangeants. En effet, il est question d'émotions intenses (Ria et Serres, 2005), d'inquiétudes (Capel, 1998), d'angoisses (Perrenoud, 2001), de souffrance (Baillauquès, 1991), de stade de survie, de panique et de malaise (Huberman, 1989), d'états affectifs anxiogènes (Ria et Gal-Petitfaux, 2001), d'une première année marquée par du stress, un sentiment de burnout, d'anxiété, de frustration et de peur (Weva, 1999).

Cet article s'articule autour de l'analyse d'histoire de vie (Pineau et Le Grand, 2007) thématique traitant de la professionnalisation d'enseignantes d'EPS débutantes et plus particulièrement des émotions ressenties au cours de la première année d'enseignement. Cette recherche propose ainsi une étude de cas à partir d'une monographie d'auto supervision (Brunelle, Coulibaly, Brunelle, Martel et Spallanzani, 1991) et d'une analyse de discours, issue d'entretiens non-directifs à visée de recherche, de deux enseignantes débutantes d'EPS.

II s'agira ainsi de mieux comprendre comment la sphère émotionnelle est mobilisée lorsqu'il s'agit de passer d'un statut d'étudiant à un statut d'enseignant titulaire et comment les retours de vacances sont des moments-clefs de ce phénomène. On pourra ainsi tenter de comprendre comment se construit dans cette sphère émotionnelle, le processus de professionnalisation pour ces enseignantes d'Éducation Physique et Sportive.

\section{1. Émotions et apprentissage: une dyade indissociable pour l'enseignant d'EPS}

1. 1. États affectifs, émotions, sentiments

L'émotion est définie comme étant une réaction intense qui n'existe pas en soi mais uniquement dans la relation à autrui (Despret, 1999). Une autre définition plus complète permet une meilleure compréhension de ce concept: "réaction subjective à l'égard du milieu, accompagnée de réponses hormonales et neurovégétatives, éprouvées en général comme agréables ou désagréables et tenues pour des réactions adaptatives qui affectent notre façon de penser" (Papalia et Olds, 1988). L'émotion ou les émotions peuvent être aussi ressenties comme étant positives ou négatives, appétitives ou aversives, perturbatrices ou facilitatrices de l'action. Ria (2007) définit les émotions comme étant constituées à la fois d'états affectifs et de sentiments. Le sujet de cette recherche est centré sur la valeur positive ou négative et non sur la catégorisation des ressentis émotionnels d'enseignantes débutantes d'EPS. Ainsi au cours de cet article, aucune 


\section{eJRIEPS 21 juillet 2010}

distinction n'est faite entre les termes émotions, affects (ou états affectifs), sentiments ainsi que tous les termes s'y rapportant.

1. 2. Des enseignants débutants en souffrance

Au regard de la littérature existante, on peut faire l'hypothèse que les débuts de carrière des enseignantes étudiées sont davantage teintés d'émotions négatives. En effet la période des débuts dans l'enseignement est vécue massivement comme un moment difficile (Baillauquès, 1990). Les enseignants débutants éprouvent des émotions intenses, des sentiments d'inconfort, de frustration, d'agacement, de déception (Ria, 2004). Les premiers pas dans l'enseignement sont plus particulièrement une source d'angoisse et de stress. Huberman (1989) révèle que l'entrée dans la carrière des enseignants a été vécue comme étant une période de survie et que 53\% d'entre eux évoquent des difficultés dans l'expérience vécue.

À leur arrivée dans un établissement, les enseignants débutants ont des difficultés multiples: une remise en question de ce qu'ils sont, les personnes d'expérience ne veulent pas aider les nouveaux, une certaine solitude dans les décisions, une autonomie pédagogique freinée... (Rayou et Van Zanten, 2004). Les modalités d'affectation ont placé les jeunes enseignants dans les situations professionnelles les plus difficiles: enseignement dans des disciplines connexes, services en lycée professionnel pour de jeunes certifiés, affectation dans les zones sensibles, fonctions de remplacement non demandées (Rapport Bancel, 1999). "Il arrive que certains stagiaires soient en souffrance (...), au point que la situation d'enseignement devient alors une source constante de peur ou d'anxiété" (Lamotte et Nérin, 2003). «Leur entrée dans le métier s'effectue souvent avec fougue et enthousiasme, mais aussi dans le doute, l'incertitude et la crainte » (Gelin, Rayou et Ria, 2007).

\section{3. Les spécificités de l'enseignement de l'EPS}

Trois principaux points permettent de différencier l'enseignement de l'EPS de celui d'autres matières : l'organisation spatiale du cours, les dogmes fondateurs transgressés et une nécessaire adaptation à l'environnement afin d'assurer la sécurité corporelle des élèves. L'organisation spatiale du cours est totalement différente dans un cours d'EPS. II a lieu dans une pièce qui n'est pas une classe où l'élève n'est pas cloisonné dans un espace, assis sur une chaise. La relation face-à-face, lieu de convergence de tous les regards représenté par l'estrade ou le tableau n'existe pas ou très peu. La distance instituée dans le champ pédagogique (Filloux, 1974) de la classe est absente. 


\section{eJRIEPS 21 juillet 2010}

Les dogmes prégnants de l'Éducation Nationale de l'école de Jules Ferry, définis par Michel Foucault et précisés par Daniel Denis comme étant fondamentalement " le silence et l'immobilité " (Gleyse et Valette, 1999) sont transgressés lors du déroulement d'une séance d'EPS. "Il est vrai que parmi toutes les matières, l'éducation physique occupe une place particulière. Par son ambiance participative et ses nombreuses interactions, ce cours est un lieu inhabituel d'expression pour les élèves. Ces caractéristiques, certes très positives, sont néanmoins susceptibles d'engendrer des désagréments supplémentaires pour les enseignants" (Dervaux, Carlier et Gérard, 2008). Le principe d'immobilité va à l'encontre de l'enseignement de l'EPS où le corps est mis en jeu à chaque instant. La gestion du bruit et du mouvement est accaparante pour l'enseignant d'EPS.

En EPS, le développement de la motricité est la finalité majeure des apprentissages. Les élèves s'y investissent corporellement. Un des paramètres lié à cet enseignement est la sécurité des élèves. En effet, "l'enseignement de l'éducation physique et sportive a toujours posé un problème spécifique de sécurité, toute activité physique et sportive exposant celui qui la pratique a des atteintes éventuelles à son intégrité physique" (B.O. du 17 mars 1994). Les apprentissages proposés aux élèves doivent garantir leur sécurité physique quelle que soit l'Activité Physique Sportive et Artistique (APSA) enseignée. L'enseignant d'EPS doit tenir compte des conditions matérielles et du caractère dangereux ou non des activités enseignées, ainsi que les consignes qu'il donne aux élèves afin de garder la maîtrise du cours. De ce fait, la vigilance de l'enseignant d'EPS est très sollicitée, au regard de la sécurité qu'il doit constamment assurer.

\section{Méthodologie}

Le thème de cet article s'articule autour de deux études de cas d'enseignantes débutantes d'EPS issues de deux recherches distinctes. La première étude est le résultat d'un travail de mémoire professionnel de PLC2 où l'enseignante concernée a effectué une réflexion autour de son évolution personnelle et professionnelle au cours de son année de stage grâce à la technique de l'auto supervision. Le deuxième sujet provient d'un échantillon d'un travail de recherche de doctorat où le récit de vie est utilisé comme méthodologie. Les similitudes trouvées au niveau des ressentis émotionnels exprimés entre ces deux enseignantes débutantes sont à l'origine de cette étude.

\section{1. Auto supervision, monographie}

L'entrée dans la recherche de l'enseignante pour l'écriture de son mémoire professionnel s'est faite par la technique de l'auto supervision pédagogique qui comme son nom 


\section{eJRIEPS 21 juillet 2010}

l'indique est une supervision faite par soi-même. Elle permet l'analyse des pratiques professionnelles (Boudot, 1996). Ce procédé facilite l'amélioration des capacités professionnelles de perception et d'intervention (Kretschmer, 1996).

Le sujet choisi est une enseignante débutante volontaire âgée de 25 ans, ayant obtenu l'Agrégation externe après un parcours diversifié (maîtrise, D.E.A.). La préparation au concours ne s'est pas faite en Institut Universitaire de Formation des Maîtres (IUFM) et elle n'a donc pas bénéficié de l'encadrement spécifique relatif à la première année de concours et n'a pas effectué de stage en établissement. Cependant, elle a un passé dans l'encadrement en club sportif. Le concours a été obtenu avec le rang de $19^{\text {ème }}$ sur 42 postes au niveau national. Les parents sont de profession libérale et employé administratif. Sa pratique sportive est de niveau national depuis 2 ans. Son premier établissement d'affectation est un collège situé dans un des quartiers difficiles de l'Académie de Montpellier classé en Réseau d'Éducation Prioritaire (REP).

Il a été demandé au sujet de coucher sur papier tout ce qui a été fait, ressenti, appris au cours de chaque journée d'enseignement et ceci pendant toute l'année de stage. Aucun minimum ni maximum d'écriture n'a été imposé. Ainsi l'enseignante stagiaire en EPS a réalisé au cours de son année de PLC2 une monographie qui a été utilisée pour cette étude et pour l'écriture de son mémoire professionnel. A la suite de chaque cours, l'enseignante d'EPS a écrit sa monographie sans contact avec l'enseignant-chercheur. L'étude s'est faite en fin d'année sur la base même de la monographie. 47 pages d'un petit cahier ont été écrites par le sujet. L'analyse de la monographie a été réalisée en fin d'année sur la base d'une analyse qualitative de discours. Un découpage thématique a été effectué. Les termes ou items se rapportant aux émotions ressenties comme "négatives" peur, stress, angoisse, anxiété, cauchemars, flipper, épuisement, triste, fatigue, - ou "positives" - plaisir, joie, bien-être, contentement, satisfaction, réussite - ont été relevés et différenciés de manière systématique.

Pour ce qui est de la validité de cette recherche, de nombreuses études ont été faites sur des "diary entries" (Clarke et Quill, 2003; Mc Mahon et MacPhail, 2007; Pope et O'Sullivan, 1998).

Les extraits tirés de l'auto supervision de cette enseignante débutante seront annotés (AuSu).

\section{2. Récit de vie}

Le deuxième sujet est âgé de 25 ans. Cette enseignante a un parcours atypique. Elle a préféré partir une année à Québec avant de passer le concours du Certificat d'Aptitude au 


\section{eJRIEPS 21 juillet 2010}

Professorat d'EPS (CAPEPS) afin de s'ouvrir culturellement. Cependant, son année de préparation au concours s'est faite en I.U.F.M., elle a donc effectué un stage. Elle a un passé dans l'animation et son niveau de pratique est, lui aussi, national. Son origine sociale est similaire à celle de la première enseignante, elle n'a passé qu'une seule fois le concours et son rang est $125^{\text {ème }}$ sur 400 .

Son établissement d'affectation est situé dans un collège de l'Académie de Paris nonclassé. Cette enseignante appartient à l'échantillon de la recherche de doctorat et après explication de la démarche scientifique, est venue faire un entretien semi-directif à visée de recherche.

La durée de l'entretien est de 38 minutes et il a été retranscrit dans son intégralité. Une analyse par le logiciel TROPES a permis de relever les items les plus courants classés dans un univers de référence. Ainsi, les items relatifs aux sentiments - univers de référence numéro 1 choisi par le logiciel — ont été relevés. Un complément d'analyse fait avec l'univers de référence numéro 2 permet de catégoriser les sentiments ressentis comme plutôt positifs - plaisir, satisfaction — des sentiments négatifs - peur, angoisse, stress - évoqués lors de l'entretien. Suite à l'enregistrement de l'entretien et à son analyse, ce sujet a été choisi comme complément à la première étude de cas aux vues des multiples similitudes relevées au niveau des ressentis émotionnels évoqués.

La méthode du récit de vie utilisée pour le doctorat est l'une des pratiques les plus courantes de la conversation ordinaire (Brun, 2003). Son but est « d'étudier un fragment particulier de réalité sociale historique, un objet social; de comprendre comment il fonctionne et comment il se transforme, en mettant l'accent sur les configurations des rapports sociaux, les mécanismes, les processus, les logiques d'action qui le caractérisent »(Bertaux, 1997).

De plus, cette approche biographique oriente le regard sur le quotidien, l'individuel et le collectif. Elle permet d'aborder des phénomènes de société en se constituant comme moyen de représentation, de mise en forme et de structuration de trajectoires de vie. Elle se donne ainsi pour tâches de donner du sens au vécu et de le rendre signifiant dans la recherche identitaire (Feldhendler, 2001).

Les extraits tirés du récit de vie seront annotés (RéVi).

Les deux méthodes d'investigations, ainsi que leurs résultats, ont été confrontés dans l'optique de tenter de mettre en évidence des analogies voire des régularités des deux discours dans le domaine des émotions ressenties ou du moins exprimées. II devrait être 
eJRIEPS 21 juillet 2010

ainsi possible de mieux connaître cette période critique de ce processus de professionnalisation du moins pour ces deux jeunes enseignantes femmes.

\section{Résultats}

3. 1. Apprendre à enseigner ... tout en enseignant

Les analyses de la monographie et du récit de vie permettent d'affirmer que ces PLC2 acquièrent des connaissances relatives à leur métier au cours de leur année de formation. Pour ce qui est des savoirs propres à l'enseignement de l'EPS, les enseignantes stagiaires de cette recherche comblent leurs lacunes en étudiant de façon précise les activités physiques qu'elles enseignent dans leurs classes tout en essayant de les faire correspondre aux finalités éducatives prescrites par les textes officiels. Cependant en début d'année les séances mises en place sont inadéquates, trop longues et peu respectées surtout d'un point de vue du timing:

AuSu: "Je pensais qu'en $3^{\circ}$ les élèves avaient déjà acquis quelques bases en badminton... ben non. La séance prévue était basée sur le placement du volant et sur le service (...). Il a fallu que je leur apprenne à tenir une raquette pour la plupart et surtout leur faire toucher le volant avec le tamis de la raquette. J'étais très déçue"

Lors de l'année de préparation au concours, les enseignantes ont accumulé des connaissances sur de multiples activités sur lesquelles elles étaient susceptibles d'être interrogées lors du concours. Mais elles se rendent compte que ces savoirs "technologiques" ne sont pas applicables tels quels. II est nécessaire de les adapter au public, à la classe, aux élèves:

Révi: "En oral 2, j'avais choisi natation, j'ai tellement bossé que j'ai eu 16 au concours. Mon premier jour à la piscine avec mes $6^{\circ}{ }^{\circ}$ 'ai eu peur mais ... peur tu vois. Moi, j'avais appris à régler problème après problème. Par exemple, un problème de respiration ou un problème de battements de jambes avec 10 ou 20 heures de pratique pour une classe de 4: Ben là je me suis retrouvée avec un gros problème. Dans la piscine, il n'y en avait pas deux qui avait les mêmes difficultés...! Que des problèmes: un avait peur, l'autre ne mettait pas la tête dans l'eau, un autre n'arrivait pas à tenir la planche, les genoux qui sortent... . Le pire a été au niveau de la communication des consignes entre les bonnets et l'eau dans les oreilles. Et dire que j'ai eu 16 en oral $2 . .$. ."

Avec l'expérience et l'avancée dans l'année scolaire les enseignantes débutantes étudiées acquièrent des savoirs et des savoir-faire et il leur est possible progressivement de les mobiliser pour faire face à une situation qu'elles ont déjà vue ou vécue :

AuSu: "En début d'année je faisais mon appel, j'expliquais la séance, donnais quelques consignes puis la première situation d'apprentissage et hop... on y allait. Mais je me suis rendue compte que 


\section{eJRIEPS 21 juillet 2010}

quand il fallait installer du matériel pour le cours du style, monter les poteaux de volley ou encore mettre en place le praticable de gym, il était plus simple de mettre le matériel en premier. Une fois l'installation mise en place les élèves ne se souvenaient absolument plus des consignes et le retour au calme une deuxième fois même pas 10 minutes après l'appel, était très difficile pour eux. Là ils mettent le matériel en place, puis ils viennent s'asseoir, je fais l'appel, j'explique puis ils y vont...."

Elles enseignent en même temps qu'elles apprennent à enseigner. Les problèmes de gestion de classe deviennent ponctuels en fin d'année scolaire. Pendant cette année de formation, elles ont appris à connaître et à gérer leurs élèves:

Révi: "(...) dans le vestiaire des garçons, il y avait un silence inquiétant... . Je ne suis pas habituée à çà, donc j'ai collé mon oreille à la porte! Et puis j'en ai entendu un, Sébastien, suggérer l'idée de pousser Thomas sous la douche. Le problème...ou plutôt son problème est que j'ai reconnu sa voix, il ne s'y attendait pas du tout (...). Et dire que j'ai évité une bêtise, juste en reconnaissant sa voix!"

Plus l'année avance et plus les enseignantes débutantes apprennent de leurs expériences sur le terrain et se construisent progressivement des connaissances professionnelles. L'activité enseignante ainsi que les savoirs professionnels s'y rattachant sont abordés lors de cette année de formation, certains plus que d'autres aux vues des priorités de ces enseignantes débutantes d'EPS. Gérer la classe, maintenir l'ordre et favoriser les apprentissages font parties de leurs priorités. Savoir comment fonctionne un établissement, travailler en équipe, se centrer sur les besoins des élèves et formaliser leur pratique professionnelle ne sont pas prépondérantes. Cependant, celles-ci se rendent bien compte qu'il leur est nécessaire d'incorporer toutes leurs connaissances afin de pouvoir les utiliser. En effet, toute expérience d'enseignement est un lieu "de mobilisation et de transformation, voire de construction, des connaissances professionnelles acquises antérieurement" (Flavier, 2003).

3. 2. Des émotions qui se succèdent mais qui ne se ressemblent pas

L'étude des corpus laisse apparaître deux types d'émotions: des émotions dites "positives" et des émotions ressenties comme "négatives". Ces dernières, en début d'année, sont tellement envahissantes qu'elles laissent peu de place aux émotions agréables. À l'inverse, les sensations de plaisir, de bien-être et de satisfaction, quasiment inexistantes en début d'année, tendent à se développer et atteignent un niveau important en fin d'année scolaire. 


\section{eJRIEPS 21 juillet 2010}

Trois périodes bien distinctes sont identifiables quant aux émotions ressenties par les deux stagiaires. La première période se situant de septembre à début décembre, laisse place aux angoisses débordantes et à un plaisir ponctuel peu ressenti:

Révi: "C'était le jour de la rentrée et j'étais tiraillée entre plusieurs sentiments: la fierté d'avoir réussi, la réalité du terrain et "qu'est ce que je fais ici?" Je ne savais absolument pas comment cela allait se passer. La seule chose que je sais, c'est que j'étais pétrifiée et que mon cœur battait à deux mille. Quand je suis rentrée chez moi, j'étais tellement sur les nerfs, que j'ai dormi 2 heures. Je n'étais même pas contente d'avoir fait mon premier jour et encore moins satisfaite de ce que j'avais fait: I'horreur."

La deuxième période allant alors jusqu'à mi-janvier est caractérisée par des angoisses dégressives alors que des émotions positives prennent place progressivement dans l'enseignement de l'EPS:

AuSu: "(...) on a rigolé toute la matinée. Même si enseigner l'escalade me stresse car j'ai toujours peur de la chute, on arrive à s'amuser souvent. Ils aiment me taquiner et se moquer de mon accent (...). Cela nous rapproche beaucoup."

Enfin, la dernière étape se déroulant jusqu'à la fin de l'année scolaire est caractérisée par des angoisses plutôt ponctuelles d'ordre pédagogique et/ou didactique mais n'altèrent en aucun cas le sentiment général de bien-être et de satisfaction:

AuSu: "Tout se passait bien jusqu'à ce moment là...j'ai ressenti une peur panique, c'était horrible. (...) mais en rentrant à la maison, j'avais oublié cet incident et j'étais quand même contente de ma séance. C'est en faisant des erreurs que l'on apprend."

Si en début d'année le plaisir ressenti par les PLC2 est plutôt ponctuel et de l'ordre du pédagogique ou du didactique, il devient important en fin d'année scolaire. Inversement, les angoisses qui sont quasi-permanentes en début d'année scolaire disparaissent pour n'être plus que ponctuelles en fin d'année scolaire.

3. 3. Des vacances oui, mais... et la reprise?

L'étude de la dynamique des émotions ressenties au cours de l'année scolaire montre une diminution progressive des émotions dites "négatives". Cependant une analyse plus précise révèle une dynamique plus complexe de cette diminution des états anxiogènes. En effet tout au long de leur année de stage, les enseignantes d'EPS se sentent de plus en plus à l'aise dans leur enseignement mais la sensation de bien-être disparaît à chaque retour de vacances. La reprise est synonyme d'angoisses, d'insomnies, de stress mais aussi de questionnement. 


\section{eJRIEPS 21 juillet 2010}

II est possible de constater un recul des émotions "positives" des deux stagiaires, la veille de la reprise ainsi que le jour de la reprise. Leur état d'anxiété est très important, elles ont du mal à s'endormir:

AuSu: "La veille de la rentrée des classes j'ai ressenti un peu l'angoisse de retrouver mes élèves (...). Depuis le retour des vacances, la nuit du lundi au mardi est un calvaire: je ne dors plus! Le cours du mardi, de $8 \mathrm{~h}$ à $10 \mathrm{~h}$ me paraît insurmontable. La classe de $5 \%$ est une classe difficile à mes yeux, avec des élèves agités, violents, peu volontaires, irrespectueux des consignes, de la sécurité mais aussi irrespectueux envers moi. Je sais que j'ai eu du mal à les cadrer la semaine précédente et j'en déduis que demain se déroulera de la même manière donc je ne trouve pas le sommeil."

Mais aussi:

RéVi: "Je n'ai pas fermé l'œil de la nuit... sans cesse j'imaginais mes séances. La veille de la rentrée des classes, j'ai ressenti un peu l'angoisse de retrouver mes élèves tu vois... pas parce que je ne les aime pas, mais... parce que j'avais peur qu'ils aient oublié ce qu'on avait mis en place avant les vacances."

La présence de l'enseignant dans la classe et avec les élèves ne va plus de soi et les explications sont peu précises, les rémédiations vagues et la volubilité n'est plus:

RéVi: "J'étais stressée et je bafouillais beaucoup alors que cela ne m'était plus arrivé depuis un moment. J'hésitais dans mes consignes, je me perdais dans mes explications (...). Ensuite, cela m'a passé mais cela a quand même duré une bonne quinzaine."

Les apparitions cycliques de ces émotions vécues comme "négatives" correspondent en tout état de cause à des périodes précises du calendrier scolaire. En effet, que ce soit d'un point de vue de l'autosupervision comme du récit de vie, chaque fin de vacances scolaires est à l'origine des réapparitions des angoisses des deux enseignantes débutantes.

\section{Discussion}

4. 1. Des enseignantes débutantes d'EPS comme les autres

Nombreux sont les articles relatifs aux enseignants débutants. Certains se focalisent sur la formation et l'accompagnement de l'entrée dans la profession (Baillauquès, 1999; Blanchard-Laville et Nadot, 2000; Chaliès et Durand, 2000). D'autres s'intéressent aux différents cycles de la vie professionnelle (Boutin, 1999; Chouinard, 1999; Gold, 2003; Hubermann, 1989) et enfin beaucoup se focalisent sur les difficultés des enseignants lors de leurs débuts dans la profession (Baillauquès, 1999) ainsi que sur leur manque de préparation au métier (Bourques, Akkari, Broyon, Heer, Gremion et Gremaud, 2007; Nadot, 2003). 
L'apprentissage du métier de ces enseignantes débutantes d'EPS est mis en avant dans cet article. Au fil de l'année scolaire les enseignantes apprennent leur métier et les difficultés rencontrées sont les mêmes que celles évoquées dans la littérature. II est d'abord question de gestion de classe comme le met en avant Esquieu (2003), de maintien de l'ordre (Chouinard, 1999) ceci grâce à la reconnaissance des voix des élèves (Canal, 2002), puis de problèmes liés à la didactique de l'activité enseignée (Gelin, Rayou et Ria, 2007) car la formation est trop théorique et pas assez pratique (Serres, Ria et Adé, 2004). II est aussi question de l'adaptation du contenu au niveau des élèves (Rayou et Van Zanten, 2004), d'improvisation (Antigny, 1994; Yinger, 1987) et d'expériences typiques débouchant à la mise en place de procédures d'interventions adéquates (Serres, Ria, Adé et Sève, 2006). De nombreux thèmes ne sont pas abordés dans cet article mais les enseignantes débutantes sont aussi confrontées à des conflits (Flavier, 2003), ressentent des frustrations (Riel, 1999) et doivent faire face à des problèmes relatifs à leur insertion professionnelle (Martineau, 2006). La revue de littérature effectuée ainsi que le traitement de l'auto-supervision et du récit de vie mettent bien en avant que ces enseignantes d'EPS débutantes sont confrontées aux mêmes problèmes que les enseignants tout degré et matière confondus.

\section{2. Des émotions présentes}

Les émotions de l'enseignant débutant sont aussi un sujet d'écriture important. Les écrits relatifs au début de carrière des enseignants sont souvent décrits comme perturbateurs, anxiogènes, stressants. Certains se sont attachés à montrer l'importance du rôle des émotions dans l'orientation concrète de l'activité de l'enseignant en classe (Ria, 2005) alors que d'autres se sont focalisés sur le stress ressenti lors des premiers pas dans la classe (Chartier, 2000; Cossette, 1999; Royer, 2001). II est aussi question d'émotions positives lors des débuts dans la carrière comme le soulignent Louvet et Baillauquès (1992) c'est-à-dire de l'enthousiasme d'avoir sa propre classe ou encore ponctuelles lorsque les élèves sont calmes ou que leurs comportements répondent à l'intention éducative de l'enseignant (Ria et collab, 2004). Cette multiplicité d'émotions ressenties se retrouve lors de l'étude de l'auto supervision ainsi que du récit de vie des deux enseignantes d'EPS.

II est aussi mis en avant que les émotions ressenties comme étant perturbatrices diminuent progressivement au cours de l'année pour laisser place à des émotions dites "positives". Cependant nous sommes face à un "vide littéraire" relatif à l'évolution des émotions des enseignants au cours de leur carrière. Une étude faite sur des enseignants 
eJRIEPS 21 juillet 2010

débutants révèle que «(...) les enseignants font référence à plusieurs problèmes (discipline, motivation des élèves, hétérogénéité, programme...) qui sont sources d'angoisse " (Heer et Akkari, 2003). II en est de même dans cette étude où, en majeure partie, les diverses difficultés auxquelles les enseignantes sont confrontées sont source de ressentis émotionnels vécus comme "négatifs". Quelques études sollicitées par I'Education Nationale s'intéressant aux parcours professionnels de jeunes enseignants français, mettent en avant que l'intensité des difficultés diminue lors de la deuxième année d'enseignement (Esquieu et Péan, 1996). II serait possible alors d'en déduire qu'avec le temps, les difficultés s'amenuisent et qu'il en est de même avec les angoisses ressenties, mais peut-on pour autant en déduire qu'avec cette diminution des difficultés, les émotions "négatives" ont aussi tendance à diminuer? En effet, Huberman (1989) met en avant le fait que certains professeurs parlent de cauchemars qu'ils faisaient et que certains font encore. Ainsi l'acquisition d'expérience n'est pas forcément synonyme de diminution des difficultés éprouvées et encore moins de ressentis émotionnels "négatifs" en baisse.

4. 3. Les vacances: période de repos, de remise à niveau ou doutes?

L'étude des stagiaires tend à montrer que certaines périodes de cette première année sont plus propices à l'apparition des émotions ressenties comme "négatives" que d'autres. Une étude plus approfondie des vacances et de l'occupation des PLC2 pendant ces vacances permet de tirer quelques conclusions intéressantes. Tout d'abord, les enseignantes débutantes d'EPS de cette recherche ont utilisé leurs vacances à de multiples fins. De manière générale, les vacances scolaires permettent aux enseignants de se reposer. Ce qu'ont fait les enseignantes stagiaires.

Cependant, les vacances leur ont permis tout d'abord d'essayer de combler leurs lacunes sur un bon nombre d'APSA mais aussi de mettre en place tous les cycles, les séances, les situations d'apprentissage prévues pour la prochaine période d'enseignement (en moyenne 6 à 8 semaines entre chaque période de vacances). Leurs vacances ont été beaucoup moins reposantes que l'on pourrait le croire...:

RéVi: "Pendant les vacances de la Toussaint j'ai pas mal bossé. Oui, j'ai préparé mes cycles avec mes projets de cycle et mes projets de classe. Mais j'ai quand même pris du temps pour moi... pour sortir un peu. Je n'ai pas fait que bosser quand même, mais bon heu... j'y ai passé pas mal de temps. J'ai d'ailleurs eu un peu l'impression de brasser du vent quoi ... ."

De nombreuses séquences sont ainsi à relever au retour des vacances. Les insomnies, les cauchemars et l'apparition des émotions "négatives" proviennent du fait que premièrement, les enseignantes d'EPS ont coupé tout contact avec les élèves et le fait de les revoir crée une tension chez elles. Elles se posent de multiples questions mais sont 


\section{eJRIEPS 21 juillet 2010}

surtout persuadées que pendant les vacances les élèves ont tout oublié. Les règles organisationnelles ont été très difficiles à mettre en place et pour les enseignantes et pour les élèves. Ainsi, la reprise est synonyme pour les élèves aux yeux des enseignantes stagiaires d'oubli inconditionnel de ces routines organisationnelles.

A ce jour, aucune étude n'apparaît sur le rôle des vacances, l'effet de ces coupures répétitives dans la professionnalisation des enseignants débutants. Cependant, "tous ou presque le disent: la première heure, le premier cours, le premier contact, dès le jour de la rentrée sont déterminants. Même les professeurs qui possèdent vingt années d'expérience, (...) confessent que les veilles de rentrée ne sauraient être, pour ce motif, des dates ordinaires" (Harmon, 1984). Gelin et collab. (2007) mettent aussi en avant le fait que "les enseignants débutants éprouvent souvent des émotions intenses en début de cours, en début d'année scolaire (...)". Il est possible d'affirmer que dans cette étude, les vacances sont ressenties comme une période de doutes où les enseignantes stagiaires se questionnent sur la reprise de contact avec leurs élèves: auront-ils tout oublié, va-t-il falloir tout ré expliquer, réinstaurer les règles, retrouver sa place au sein du groupe, se faire respecter, les remettre au travail...? Ainsi, le retour des vacances est vécu émotionnellement à chaque fois par ces deux enseignantes comme une nouvelle rentrée où tout semble à (re)faire, à (re)construire avec les élèves.

\section{4. Les spécificités de l'EPS}

Le support d'enseignement de l'EPS c'est-à-dire les APSA, sont aussi des éléments de différenciation de l'EPS des autres matières dites nobles. En effet, maitrise et dangerosité de l'activité enseignée sont aussi sources de ressentis émotionnels spécifiques chez les deux enseignantes débutantes étudiées.

\section{4. 1. La maîtrise de l'activité}

II est aisé de comprendre que les enseignantes débutantes doivent, lors du changement d'APSA, tout reprendre du début et de nouveau acquérir des connaissances relatives à cette activité: le règlement, les différentes formes de pratique, les représentations des élèves, les divers objectifs à atteindre, les multiples difficultés rencontrées par les élèves, les situations d'apprentissage possibles, l'aménagement du matériel.... En effet, " particulièrement en E.P.S., la multiplicité des objets de savoirs susceptibles d'avoir à être enseignés ne peut pas véritablement avoir été rencontrée par l'enseignant, compte tenu de la diversité des APSA constituant le champ social." (Buznic-Bourgeacq, Terrisse et Margnes, 2004). Le fait de ne pas connaître l'activité enseignée ou de ne pas savoir comment l'enseigner est source d'angoisse pour ces débutantes: 


\section{eJRIEPS 21 juillet 2010}

AuSu: "Je déteste la natation, non pas que j'ai peur de l'eau, mais je ne connais rien à la logique de cette APSA (mis à part qu'il ne faut pas se noyer). Je ne sais pas comment je vais devoir me comporter quand je serai au bord du bassin et eux dans l'eau. (...) Au bord du bassin, j'ai le cœur qui va exploser! Je les fais passer deux par deux, je suis accrochée à la perche et je ne les quitte pas des yeux."

Il en est de même quant à la mise en place des moyens didactiques nécessaires à l'enseignement de l'activité:

RéVi: "(...) le stress était important pour le nouveau cycle et surtout comment le mettre en place avec les terrains, le nombre d'élèves présents, quelles réactions ils auraient à l'aménagement de certaines règles etc."

Le rapport au savoir est totalement différent selon que l'activité est connue et maîtrisée ou pas. En effet, ces mêmes auteurs (Buznic-Bourgeacq et collab., 2004) démontrent qu'une enseignante débutante d'EPS forte d'une histoire singulière, d'expériences uniques, est marquée d'un rapport spécifique aux savoirs qu'elle a la charge d'enseigner. L'approche et l'enseignement sont différents selon la maîtrise de l'activité physique. Ainsi, il est à même de se demander si le ressenti émotionnel "négatif" des enseignantes d'EPS au retour des vacances n'est pas la conséquence de l'enseignement de certaines activités nonmaîtrisées car pour des raisons purement pratiques et organisationnelles, les établissements scolaires d'accueil des deux PLC2 proposent des cycles de pratiques sportives allant de vacances à vacances.

4. 4. 2. La dangerosité de l'activité enseignée

La sécurité corporelle de l'élève étant une condition sine qua non de l'enseignement de l'EPS, le caractère dangereux de l'activité est un facteur primordial des émotions négatives ressenties:

AuSu: "Premier jour d'escalade avec mes 5\%. J'ango issais réellement. J'ai eu beaucoup de difficultés à rester sereine pendant ces deux semaines de vacances rien qu'à l'idée que j'allais avoir deux activités à risques pendant ce troisième cycle avec "MA" classe difficile (lutte et escalade). Je suis arrivée en cours très tendue, après avoir imaginé les pires scénarii pendant quinze jours. Pourtant je savais que je maîtrisais cette activité et que je n'aurais pas de difficulté à l'enseigner. Le problème résidait non pas dans l'enseignement, mais dans l'encadrement, la gestion de la sécurité. J'avais une peur bleue de laisser certains élèves en assurer d'autres."

Cet extrait illustre bien le ressenti émotionnel lors de son enseignement dans une APSA dite à risques. Malgré sa connaissance de l'activité, le caractère dangereux de cette activité prend le dessus sur les compétences de cette enseignante. Le récit de vie et l'autosupervision fournissent de nombreux extraits relatifs à la dangerosité de l'activité enseignée. 
La professionnalisation de ces enseignantes d'EPS semble différente de celle des autres enseignants. Les APSA sont le support de l'EPS, et le fait de ne pas les maîtriser est un facteur anxiogène important. Sans maîtrise de l'activité, les stagiaires s'exposent à une mauvaise gestion de la classe, des consignes et ne parviennent pas à évaluer les dangers potentiels spécifiques à l'enseignement de l'APSA. Avoir des lacunes sur une partie du programme n'engendre pas les mêmes conséquences dans les autres matières qu'en EPS où l'intégrité physique des élèves doit être préservée.

Plus l'activité proposée est dangereuse, plus les risques de manquement à la sécurité sont nombreux, plus l'intégrité physique de l'élève est menacée, plus l'état anxiogène prévaut. Veiller à et assurer la sécurité corporelle de chaque élève fait parti du devoir de l'enseignant et engendre ainsi une professionnalisation différente de celle des PLC2 des matières "intellectuelles". Cette gestion sécuritaire est source de ressentis émotionnels "négatifs" pour ces stagiaires étudiées.

Dans cette étude, les facteurs dangerosité et/ou maîtrise de l'activité sont importants et sont à l'origine de nombreuses émotions ressenties comme négatives et en font une spécificité de la professionnalisation de ces enseignantes débutantes d'EPS.

\section{Conclusion}

Cette étude a permis de mettre en avant la spécificité de la professionnalisation de deux enseignantes stagiaires d'EPS au vu de leurs ressentis émotionnels au cours de la première année d'enseignement. Les émotions sont multiples, perçues comme "négatives" en début d'année et présentant une dynamique au cours de l'année scolaire en fonction de l'itération des vacances scolaires et des caractéristiques de I'APSA enseignée. Leur professionnalisation présente de nombreuses similitudes avec celle des autres enseignants, liées aux difficultés de gestion de classe, l'organisation de la séance et l'apprentissage des élèves. Toutefois, l'enseignement d'APSA non-maîtrisée ainsi que leur caractère dangereux sont des contraintes peu présentes ailleurs.

Les coupures dues aux vacances jouent aussi un rôle dans le processus de professionnalisation des deux stagiaires. La question qui se pose alors est de savoir si les angoisses ressenties par ces enseignantes débutantes lors de la reprise sont juste la cause des vacances et/ou la cause du changement de l'activité physique enseignée. Une étude plus approfondie est nécessaire afin de différencier les ressenties émotionnels "négatifs" dus aux vacances et ceux dus aux spécificités de l'activité enseignée. 


\section{eJRIEPS 21 juillet 2010}

Les enseignantes débutantes décrites dans cet article ont permis de relever l'importance des émotions ressenties au cours de cette année scolaire de formation. Cependant, ce n'est pas tant la situation qui était stressante mais plutôt l'évaluation de la situation par l'enseignant débutant et la signification de cette même situation qui engendrait du stress chez ces enseignantes. Cossette (1999) et Gendron (2006) mettent en avant les nombreuses différences entre les enseignants hommes et femmes au niveau du stress ressenti.

Cette étude s'est attachée à mettre en avant les émotions ressenties en début de carrière pour des enseignantes débutantes d'EPS. La question se pose alors de savoir si le fait d'avoir étudié le cas de deux femmes n'altère pas les résultats au plan des émotions relevées. En effet il est possible de se demander si les émotions ressenties par ces stagiaires femmes sont identiques à celles ressenties par des enseignants masculins ou très spécifiques. Selon l'autoévaluation de ses propres compétences professionnelles, l'enseignant homme va-t-il percevoir la maîtrise et/ou le caractère dangereux de l'activité physique enseignée de la même manière que si c'était une enseignante femme? La dynamique émotionnelle mise à jour dans cette étude sera-t-elle différente et indépendante des vacances et des caractéristiques spécifiques des APSA enseignées dans le cadre d'une étude d'enseignants débutants d'EPS hommes ?

\section{Bibliographie}

Antigny, S. (1994). Le nouvel enseignant face à sa classe. Éducation et formations, 37, 89-94.

Baillauques, S. (1991). La prise de fonction des enseignants: un faux rite d'initiation?

Débuter dans le métier d'enseigner, Cahiers pédagogiques, 290, 18-23.

Baillauques, S. (1999). Ce que l'entrée dans la carrière révèle du rapport des enseignants à la formation. In J.-C. Hétu, M. Lavoie et S. Baillauquès (Dir.), Jeunes enseignants et insertion professionnelle. Un processus de socialisation? De professionnalisation? De transformation? Bruxelles, Belgique: De Boeck Université.

Bancel, D. (1999). Rapport au ministre sur les conditions de travail et de vie des enseignants de lycée.

Bertaux, D. (1997). Les récits de vie. Paris: Editions Nathan.

Blanchard-Laville, C., et Nadot, S. (2000). Malaise dans la formation des enseignants. 
Paris : L'Harmattan.

Boudot, M.-F. (1996). La supervision: une relation formative. In C. Blanchard-Laville et D. Fablet (Dir), L'analyse des pratiques professionnelles. Paris : L'Harmattan.

Bourque, J., Akkari, A., Broyon, M.-A., Heer, S., Gremion, F., \& Gremaud, J. (2007). L'insertion professionnelle des enseignants: recension d'écrits. In A. Akkari, L. Solar-Pelletier \& S. Heer (Dir.), L'insertion professionnelle des enseignants. Bienne : Editions HEP-BEJUNE.

Boutin, G. (1999). Le développement de l'identité professionnelle du nouvel enseignant et l'entrée dans le métier. In J.-C. Hétu, M. Lavoie et S. Baillauques (Dir.), Jeunes enseignants et insertion professionnelle. Un processus de socialisation? De professionnalisation? De transformation? Bruxelles : De Boeck Université.

Brun, P. (2003). Le récit de vie dans les sciences sociales. La revue trimestrielle $d u$ mouvement ATD quart Monde, dossier 188, «l'écriture de la vie ».

Brunelle, J., Coulibaly, A., Brunelle, J. P., Martel, D., et Spallanzani, C. (1991). La supervision pédagogique. Revue EPS, 227, 58-64.

Buznic-Bourgeacq, P., Terrisse, A., et Margnes, E. (2007). L'expérience personnelle du professeur d'EPS, approche clinique et implications didactiques: une étude de cas d'une enseignante débutante. eJRIEPS, 11, 20-38.

Canal, J.-L. (2002). Ethnographie d'une classe ordinaire de $6^{\circ}$ en EPS : L'épreuve des limites. Thèse de doctorat inédite, UFR STAPS, Montpellier.

Capel, S. (1998). Experiences of Physical Education students in learning to teach. European Physical Education Review, 4, 127-144.

Chalies, S., et Durand, M. (2000). Note de synthèse. L'utilité discutée du tutorat en formation initiale des enseignants. Recherche et formation, 35, 145-180.

Chartier, J. (2000). Angoisse, prévention et prise de risques. In C. Blanchard-Laville et S. Nadot (Dir.), Malaise dans la formation des enseignants. Paris : L'Harmattan.

Chouinard, R. (1999). Enseignants débutants et pratiques de gestion de classe. Sciences de l'éducation, 3(25), 497-514.

Clarke, G., et Quill, M. (2003). Researching sport education in action: a case study. European Physical Education Review, 9(3), 253-266.

Cossette, F. (1999). Les sources de stress, le stress ressenti et le soutien social chez les enseignants en début de carrière. Thèse de doctorat inédite, Université du Québec, Montréal.

Dervaux, P., Carlier, G., et Gérard, P. (2008). Regards croisés des stagiaires et des 
élèves sur l'origine d'incidents disciplinaires significatifs au cours d'éducation physique. eJRIEPS, 13, 26-40.

Despret, V. (1999). Ces émotions qui nous fabriquent, Paris : Seuil.

Doyle, W. (1986). Classroom and management. Dans M. C. Wittrock (Dir.), Handbook on Research on Teaching, 3e édition. New-York: Mac Millan.

Esquieu, N. (2003). Être professeur en collège et lycée en 2002. Note d'information, 03.37(Juin).

Esquieu, N., et Péan, S. (1996). Les débuts dans le métier des enseignants du second degré. Bilan des deux premières années d'exercice. Direction de l'évaluation et de la perspective, Octobre 1996 (Note d'information 96.42).

Feldhendler, D. (2001). Approche dramatique du récit de vie : une démarche interculturelle. Actes de $\mathrm{VII}^{\circ}$ Congrès de l'Association pour la recherche interculturelle (ARIC).

Filloux, J. (1974). Du contrat pédagogique. Paris : Bordas.

Flavier, E. (2003). La formation des enseignants débutants en EPS: la participation des situations conflictuelles au processus de construction des connaissances professionnelles. eJRIEPS, 3, 40-50.

Gelin, D., Rayou, P., et Ria, L. (2007). Devenir enseignant. Parcours et formation. Paris : Armand Colin.

Gendron, B. (2006). Capital émotionnel et genre: ce capital qui fait aussi la différence entre les filles et les garçons à l'école et au travail. Cahier de la maison des Sciences Economiques, Série rouge, n76.

Gleyse, J., et Valette, M. (1999). Rites initiatiques et rituels de passage ou de purification dans l'école, L'EP et le sport. In Gleyse J. (Dir.): Corps, sports et rites (4). Montpellier : Corps et culture.

Gold, P. W. (2003). Mentor and protégé attitudes toward secondary level mentoring programs in Suffolk County. Dowling College, New York.

Hamon, H., et Rotman, P. (1984). Tant qu'il y aura des profs. Paris : Seuil.

Heer, S., et Akkari, A. (2004). Les débuts dans le métier d'enseignant: le bilan des premières années d'exercice. In S. Heer et A. Akkari (Dir.), Recherches sur l'enseignement, les didactiques disciplinaires et la formation des enseignants: regards croisés: Actes du Forum Recherches 2003 de la HEP-BEJUNE.

Huberman, M. (1989). La vie des enseignants: Évolution et bilan d'une profession. Lausanne : Delachaux et Niestlé. 


\section{eJRIEPS 21 juillet 2010}

Kretschmer, M. (1996). A propos de la supervision, une réflexion multiple. In C. BlanchardLaville et D. Fablet (Dir.), L'analyse des pratiques professionnelles. Paris : L'Harmattan.

Lamotte, V., et Nerin, J.-Y. (2003). Le CAPEPS : se préparer aux épreuves écrites et orales (Vol. 61). Paris : Revue EPS.

Lamour, H. (1986). Traité thématique de pédagogie de l'EPS. Paris : Vigot.

Louvet, A., \& Baillauquès, S. (1992). La prise de fonction des instituteurs. Paris : INRP.

Martineau, S. (2006). Chronique sur l'insertion professionnelle en enseignement. A propos de l'insertion professionnelle en enseignement. Formation et Profession (Avril 2006), 48-53.

Ministère de l'Éducation Nationale (1994). La sécurité. B. O. №11 du 17 mars 1994.

Ministère de l'Éducation Nationale (2002). Livret national du professeur stagiaire.

McMahon, E., et MacPhail, A. (2007). Learning to teach sport education: The experiences of a pre-service teacher. European Physical Education Review, 13, 229-246.

Nadot, S. (2003). L'entrée dans le métier d'enseignant ou la pratique à ses débuts. In G. Boutin (Dir). La formation des enseignants en question. Montréal : Éditions Nouvelles.

Papalia, D. E., et Olds, S. W. (1988). Introduction à la psychologie. Montréal : Mac Graw Hill.

Perrenoud, P. (2001). Développer la pratique réflexive dans le métier d'enseignant. Paris : ESF.

Pineau, G. et Le Grand J.-L. (2007) Les histoires de vie, Que Sais-je? 4e édition, Paris : PUF.

Pope, C., et O'Sullivan, M. (1998). Culture, pedagogy and teacher change in an urban high school: How would you like your eggs done? Sport, education and society, 3 , 201-236.

Rayou, P., et Van-Zanten, A. (2004). Enquête sur les nouveaux enseignants : changerontils l'école ? Paris : Bayard.

Ria, L., et Gal-Petitfaux, N. (2001). Apprendre à enseigner l'éducation physique. In M. Récopé (Dir.), L'apprentissage. Paris : Edition Revue EP.S.

Ria, L., Sève, C., Durand, M., et Bertone, S. (2004). Indétermination, contradiction et exploration: trois expériences typiques des enseignants débutants en éducation physique. Revue des sciences de l'éducation, 30(3), 535-554.

Ria, L. (2005). Les émotions. Paris : Éditions revue EPS. 


\section{eJRIEPS 21 juillet 2010}

Ria, L., et Serres, G. (2005). Formation des professeurs-stagiaires: apprendre un métier en alternance. Revue EPS, 315, 63-65.

Ria, L. (2007). Autour des mots "émotion" et "plaisir". In P. Gagnaire et F. Lavie (Dir.), Le plaisir des élèves en EPS. Futilité ou nécessité. Géménos: Coédition AEEPS et AFRAPS.

Riel, R. (1999). L'art d'être jeune prof. Vie pédagogique, 111(Avril-mai), 10-12.

Roux-Perez, T. (2006). Les enseignants néo-titulaires à l'épreuve du métier. Les langues modernes, 100(3), 34-44.

Royer, N., Loiselle, J., Dussault, M., Cossette, F., et Deaudelin, C. (2001). Le stress des enseignants québécois à diverses étapes de leur carrière. Vie pédagogique, 119, 5-8.

Serres, G., Ria, L., et Adé, D. (2004). Modalités de développement de l'activité professionnelle au gré des contextes de classe et de formation: le cas des professeurs stagiaires en Education Physique et Sportive. Revue Française de Pédagogie, 149, 49-64.

Serres, G., Ria, L., Adé, D., et Sève, C. (2006). Apprend-on vraiment à intervenir en formation initiale? Prémisses du développement de l'activité professionnelle dans les dispositifs de formation en alternance. STAPS, 72(2), 9-20.

Yinger, J. R. (1987). By the seat of your pants: An inquiry into improvisation and teaching. Paper presented at the annual conference of the American Educational Research Association (AERA). Washington, DC.

Weva, K. (1999). Insertion professionnel des nouveaux enseignants: responsabilité de l'administration scolaire. Dans J.-C. Hétu, M. Lavoie et S. Baillauques (Dir.), Jeunes enseignants et insertion professionnelle. Un processus de socialisation? De professionnalisation? De transformation? Bruxelles : De Boeck Université. 